\title{
Output Consensus of Heterogeneous Multiagent Systems with Physical and Communication Graphs
}

\author{
Junwei Wang $\left(\mathbb{D},{ }^{1}\right.$ Kairui Chen $\mathbb{D}^{2}{ }^{2}$ and Qiuli Liu $\mathbb{D I}^{3}$ \\ ${ }^{1}$ School of Mathematics and Statistics, Guangdong University of Foreign Studies, Guangzhou 510006, China \\ ${ }^{2}$ School of Automation, Guangdong University of Technology, Guangzhou 510006, China \\ ${ }^{3}$ School of Mathematical Sciences, South China Normal University, Guangzhou 510631, China
}

Correspondence should be addressed to Junwei Wang; wangjwlj@gmail.com

Received 25 May 2018; Revised 8 August 2018; Accepted 13 August 2018; Published 1 November 2018

Academic Editor: Marcelo Messias

Copyright (c) 2018 Junwei Wang et al. This is an open access article distributed under the Creative Commons Attribution License, which permits unrestricted use, distribution, and reproduction in any medium, provided the original work is properly cited.

This paper deals with the output consensus problem of heterogeneous networks in a leader-follower manner that are interconnected by a physical coupling graph. The network under consideration consists of linear agents with different dynamics/ dimensions. Both the state-feedback and output-feedback control protocols based upon information flow prescribed by a separate communication graph are developed, using the internal model principle and relative outputs of neighboring agents. With the small-gain theorem, we convert the consensus problem into a $H_{\infty}$ control problem of decoupled linear systems having the same dimensions as a single agent, where the disturbance attenuation constraints depend on the largest singular value related to the global information of physical and communication graphs. Then, we provide local synthesis procedures for control gains in terms of feasible solutions of algebraic Riccati equations. Finally, simulation examples are presented to verify the performance of the theoretical results.

\section{Introduction}

In the past decade, consensus (including leaderless consensus and leader-follower consensus) has become a hot topic due to broad applications of cooperative control of multiagent systems (MASs) [1-4]. It is concerned with designing control protocols for each agent in the presence of limited information exchanges such that eventually the group reaches an agreement on certain quantities of interest. Early consensus works focus on MASs with very primitive individual agent dynamics (such as single integrators or double integrators) [5-8], where only the information exchange among agents determines time evolution of the networks. Starting from them, the MASs with finite-dimensional linear dynamics have enticed intensive attention from the control community, which are more general and include integrator dynamics as a special case [9-13].

In the past literature of consensus, it is commonly assumed that all agents in a MAS are modeled by identical linear equations, which is said to be homogeneous networks.
In such MASs, control protocols are designed upon either relative states, relative observer states, or relative output measurements of neighboring agents to produce state consensus (see $[11,14,15]$ and references therein). However, in practice, the dynamics of individual agents are hardly exactly identical. For these MASs (often formulated as heterogeneous networks), previous control protocols for homogeneous networks not only fail to guarantee the stability of state consensus but also make state consensus meaningless in the case that the state-space dimensions of individual agents are different. Therefore, recent interest has shifted to output consensus of heterogeneous MASs, where all agents reach an agreement on their partial-state outputs through relative output information exchange with neighboring agents [16-26].

Within the literature addressing the output consensus problem of heterogeneous MASs, two approaches are of particular interest: the feedforward approach and the internal model approach. In the feedforward approach, a state or output feedback protocol is designed based on the solution of the 
output regulator equations [19]. More recently, the internal model principle has been extended to the study of output consensus control [20-23] and output containment control [27] of heterogeneous networks.

However, most of previous works are restricted to MASs interconnected by a single communication topology. However, coordination problems encountered in the cyberphysical systems (e.g., power generators and robot teams) can often be rephrased as consensus problems in which two interaction topologies coexist, i.e., the physical coupling topology and the communication topology [28, 29]. When that is the case, the first physical coupling topology connects the dynamics of the agents $[30,31]$ and then control protocols are computed using the information flow prescribed by the second communication topology $[32,33]$. To our best knowledge, the very few works that explicitly address the consensus problems of linear MASs with more than one interaction topologies are reported in [34, 35]. In [34], only the homogeneous dynamics of agents are considered. Although Lombana and di Bernardo [35] has investigated the heterogeneous case, the agents are required to have identical dimension. Furthermore, the input of each agent should have the order as many as that of the agent itself.

To fill up the abovementioned gaps, this paper considers the distributed control problem of linear heterogeneous MASs coupled by a physical coupling graph $\mathscr{G}^{\mathrm{P}}$, which is in general more difficult than that of homogeneous MASs. The control protocols are designed upon the allowed information flow prescribed by a separate communication graph $\mathscr{G}^{\text {c }}$. Compared to existing methods, the salient features of this paper are summarized as follows.

(1) We study linear MASs in a generalized environment in the sense that they have not only physical couplings between their dynamics (captured by the physical coupling graph $\mathscr{G}^{\mathrm{P}}$ ) but also a second communication graph $\mathscr{G}^{\mathrm{c}}$ allowing information flow for the design of control protocols. Furthermore, the agents have their own individual dynamics. Thus, the resulting multiagent systems are heterogeneous and coupled by multiplex interaction topologies

(2) Relying on the relative outputs between neighboring agents, both the state-feedback and output-feedback protocols are proposed. In each protocol, a dynamic compensator is constructed by the internal model principle

(3) The key element of our approach for the analysis of leader-follower output consensus is the smallgain theorem, which allows us to recast the original consensus problem as the $H_{\infty}$ control problem of a set of decoupled linear systems with the same dimensions as a single agent

(4) The decomposed $H_{\infty}$ control problem consists of both the state-feedback and output-feedback cases, with a disturbance attenuation constraint depending on the largest singular value related to the global information of physical and communication graphs.
Accordingly, the control gains can be determined by solving the algebraic Riccati equations

The rest of this paper is organized as follows. In Section 2, the notation used throughout the paper is given and the output consensus problem is formulated. Sections 3 and 4 are devoted to the main results for the state-feedback control and output-feedback control, respectively, where the global sufficient condition and local design procedures are presented. In Section 5, numerical simulations are presented to validate the main results of the paper. Section 6 concludes the paper.

\section{Preliminaries and Problem Formulation}

Let $\mathbb{R}$ and $\mathbb{R}^{n \times n}$ stand for the sets of real numbers and $n \times n$ real matrices, respectively. $j \mathbb{R}$ denotes the imaginary axis. $I_{n}$ and $1_{n}$ represent an identity matrix of dimension $n$ and a vector in $\mathbb{R}^{n}$ with elements being all ones, respectively. $\|\cdot\|$ denotes the Euclidean vector norm or the corresponding induced matrix 2-norm. For symmetric matrices $X$ and $Y$, $X \geq Y$ (respectively, $X>Y$ ) means that $X-Y$ is positive semidefinite (respectively, positive definite). diag $\{\ldots\}$ defines a block-diagonal matrix. For two matrices $X$ and $Y$, $X \otimes Y$ denotes the Kronecker product and $\bar{\sigma}(X)$ is the maximum singular value of $X$. If not explicitly stated, matrices are assumed to have compatible dimensions.

2.1. Graph Theory. A weighted directed graph (digraph) is denoted by $\mathscr{G}=(\mathscr{V}, \mathscr{E})$, where $\mathscr{V}=\{1, \ldots, N\}$ denotes the set of nodes and $\mathscr{E} \subseteq \mathscr{V} \times \mathscr{V}$ denotes the set of edges. A directed edge in $\mathscr{E}$ is denoted by the ordered pair of nodes $(j, i)$, meaning that node $i$ can receive information from node $j$. The set of neighbors of node $i$ is denoted by $N_{i}=\{j \in \mathscr{V}$ $:(j, i) \in \mathscr{E}\}$. No self-loop is allowed; hence, $a_{i i}=0, \forall i \in \mathscr{V}$. The adjacency matrix $G=\left[g_{i j}\right] \in \mathbb{R}^{N \times N}$ associated with the digraph $\mathscr{G}$ is defined by $g_{i j}>0$ if $(j, i) \in \mathscr{E}$ and $g_{i j}=0$ otherwise. The Laplacian matrix $L=\left[l_{i j}\right] \in \mathbb{R}^{N \times N}$ is defined as $l_{i i}=\sum_{k \in N_{i}} g_{i k}$ and $l_{i j}=-g_{i j}$ for all $j \neq i$. A path on the digraph is an ordered set of distinct nodes $\left(i_{1}, \ldots, i_{k}\right)$ such that $\left(i_{q-1}, i_{q}\right) \in \mathscr{E}$. A digraph is said to have a spanning tree if there exists a node, called the root, which has a directed path to every other node in the graph.

2.2. Problem Formulation. We consider a heterogeneous group with $N$ followers and a leader, in which the followers are labeled as agents $1, \ldots, N$ and the leader is labeled as agent 0 . Motivated by the applications of distributed control in cyber-physical systems, we model the interaction topology between the $N$ followers by two digraphs: one is the physical coupling digraph $\mathscr{G}^{\mathrm{p}}=\left(\mathscr{V}, \mathscr{E}^{\mathrm{p}}\right)$ with the adjacency matrix $G^{\mathrm{p}}=\left[g_{i j}^{\mathrm{p}}\right] \in \mathbb{R}^{N \times N}$ and Laplacian matrix $L^{\mathrm{p}}=\left[l_{i j}^{\mathrm{p}}\right] \in \mathbb{R}^{N \times N}$ and the other is the communication digraph $\mathscr{G}^{\mathrm{C}}=\left(\mathscr{V}, \mathscr{E}^{\mathrm{C}}\right)$ with the adjacency matrix $G^{\mathrm{c}}=\left[g_{i j}^{\mathrm{c}}\right] \in \mathbb{R}^{N \times N}$ and Laplacian matrix $L^{\mathfrak{c}}=\left[l_{i j}^{\mathcal{C}}\right] \in \mathbb{R}^{N \times N}$. 
The dynamics of $i$ th follower is given by

$$
\left\{\begin{array}{l}
\dot{x}_{i}=A_{i} x_{i}+B_{i} u_{i}+D_{i} \sum_{j \in N_{i}^{p}} g_{i j}^{\mathrm{p}}\left(y_{j}-y_{i}\right), \\
y_{i}=C_{i} x_{i}, i \in \mathscr{V}
\end{array}\right.
$$

where $x_{i} \in \mathbb{R}^{n_{i}}$ is the state, $y_{i} \in \mathbb{R}^{q}$ the measured output, $u_{i} \in \mathbb{R}^{m_{i}}$ is the control input which will be designed based on the communication graph $\mathscr{G}^{\mathrm{c}}, g_{i j}^{\mathrm{p}}$ is the element of $\mathscr{G}^{\mathrm{p}}$, and $N_{i}^{\mathrm{p}}$ is the set of neighbors of node $i$ corresponding to the physical coupling graph $\mathscr{G}^{\mathrm{p}} . A_{i}, B_{i}, C_{i}$, and $D_{i}$ are the known real constant matrices of appropriate dimensions describing the nominal dynamics of follower $i$. To avoid redundancy of measurement, it is assumed that $C_{i}$ has full row rank, i.e., $\operatorname{rank}\left(C_{i}\right)=q$.

The leader 0 which acts as an exosystem that generates a reference trajectory for the group obeys the dynamic model.

$$
\left\{\begin{array}{l}
\dot{x}_{0}=A_{0} x_{0} \\
y_{0}=C_{0} x_{0}
\end{array}\right.
$$

where $x_{0} \in \mathbb{R}^{n_{0}}$ and $y_{0} \in \mathbb{R}^{q}$ denote the state and measured output, respectively. We assume that the leader does not receive any information from followers and can be observed only from a small subset of followers. To depict whether the followers are connected to the leader, we define a leader adjacency matrix $G_{0}=\operatorname{diag}\left\{g_{10}, \ldots, g_{N 0}\right\} \in \mathbb{R}^{N \times N}$, where $g_{i 0}>0$ if the $i$ th follower obtains information from the leader 0 and $g_{i 0}=0$ otherwise.

Remark 1. Note that MAS (1) and (2) is called heterogeneous because each agent has its own distinct dynamics and their dimensions are also nonidentical. Attempts to address the distributed control problems for such systems have been addressed [19-23]. Unfortunately, the aforementioned schemes ignore the coupling effect of the originally existed physical coupling graph, thus cannot be straightforwardly applied to the considered control problem. This renders the contribution of this paper with respect to previous works considerable.

Throughout this paper, we shall use the following concept of output consensus for the heterogeneous MAS (1) and (2).

Definition 1. The heterogeneous MAS (1) and (2) coupled by the physical coupling graph $\mathscr{G}^{\mathrm{p}}$ is said to reach output consensus if for all $i \in \mathscr{V}$, there exist distributed control laws $u_{i}$ relying on the communication graph $\mathscr{G}^{\mathrm{c}}$ such that the following local output error:

$$
e_{i}(t) \triangleq y_{i}(t)-y_{0}(t) \rightarrow 0 \text { as } t \rightarrow \infty .
$$

The aim of this work is to solve the output consensus problem of the MAS (1) and (2) under the physical coupling graph $\mathscr{G}^{\mathrm{P}}$ by designing the control protocols $u_{i}$. More specifically, we consider two cases: (1) the state-feedback case in which the full state information of each follower can be measured and (2) the output-feedback case in which only partial state information of each follower is measured. To solve the aforementioned control problem, we need to introduce the concept of the internal model [36], which will help us define the dynamic compensator in the process of distributed control design.

Definition 2. A pair of matrices $\left(S_{1}, S_{2}\right)$ is said to incorporate a $q$-copy internal model of matrix $A_{0}$ if

$$
\begin{aligned}
& S_{1}=\operatorname{diag}\{\underbrace{\alpha_{1}, \ldots, \alpha_{q}}_{q \text {-tuple }}\}, \\
& S_{2}=\operatorname{diag}\{\underbrace{\theta_{1}, \ldots, \theta_{q}}_{q \text {-tuple }}\},
\end{aligned}
$$

where $\left(\alpha_{i}, \theta_{i}\right)(i=1, \ldots, q)$ are controllable and the minimal polynomial of $A_{0}$ divides the characteristics polynomial of $\alpha_{i}$.

We conclude this section by introducing some assumptions that will be required to derive the main results of this paper.

Assumption 1. The pair $\left(A_{i}, B_{i}\right)$ is stabilizable, and the pair $\left(A_{i}, C_{i}\right)$ is detectable.

Assumption 2. $A_{0}$ has no eigenvalues with negative real parts.

Assumption 3. The communication graph $\mathscr{G}^{\mathrm{c}}$ contains a spanning tree, and at least one root node in $\mathscr{G}^{\mathrm{c}}$ can observe information from the leader.

Assumption 4. For all $\lambda$ that are the eigenvalues of $A_{0}$ :

$$
\operatorname{rank}\left[\begin{array}{cc}
A_{i}-\lambda I & B_{i} \\
C_{i} & 0
\end{array}\right]=n_{i}+q
$$

\section{The Case of State-Feedback Design}

In this section, we consider the output consensus problem of MAS (1) and (2) using the state-feedback control of the form:

$$
\left\{\begin{array}{l}
u_{i}=K_{x_{i}} x_{i}+K_{z_{i}} z_{i} \\
\dot{z}_{i}=S_{1 i} z_{i}+S_{2 i} e_{i v}
\end{array}\right.
$$

where $z_{i} \in \mathbb{R}^{s_{i}}$ represents the internal controller state and $K_{x_{i}}$ and $K_{z_{i}}$ are the state-feedback gain and feedforward gain to be designed, respectively. $S_{1 i}$ and $S_{2 i}$ are constant matrices such that the pair $\left(S_{1 i}, S_{2 i}\right)$ contains a $q$-copy 
internal model of $A_{0}$ as defined in Definition 2. $e_{i v}$ is the neighborhood output error of follower $i$ defined by

$$
e_{i v}=\frac{1}{\sum_{j \in N_{i}^{c}} g_{i j}^{\mathrm{c}}+g_{i 0}}\left[\sum_{j \in N_{i}^{\mathrm{c}}} g_{i j}^{\mathrm{c}}\left(y_{i}-y_{j}\right)+g_{i 0}\left(y_{i}-y_{0}\right)\right] \text {, }
$$

where $g_{i j}^{c}$ are elements of $G^{c}$ and $N_{i}^{c}$ is the set of neighbors of node $i$ corresponding to the communication graph $\mathscr{G}^{\mathrm{c}}$.

The output consensus problem will be solved using the state-feedback control law (6) in two steps. First, a global sufficient condition for output consensus is provided by applying the output regulation technique [36], and we next present detailed local design procedures for control gains $K_{x_{i}}$ and $K_{z_{i}}$ based on the small-gain theorem [37] and the solution of $H_{\infty}$ control problems [38].

\subsection{Global Condition for Output Consensus. Denoting}

$$
\begin{aligned}
\beta & =\left[\beta_{1}^{\mathrm{T}}, \ldots, \beta_{N}^{\mathrm{T}}\right]^{\mathrm{T}}(\beta=x, e, z), \\
x_{l} & =\left[x^{\mathrm{T}}, z^{\mathrm{T}}\right]^{\mathrm{T}}, \\
\tilde{x}_{0} & =1_{N} \otimes x_{0}, \\
\tilde{\Phi} & =\operatorname{diag}\left\{\Phi_{i}\right\}\left(\Phi=A, B, C, D, K_{x}, K_{y}, K_{z}, S_{1}, S_{2}\right), \\
\tilde{A}_{0} & =I_{N} \otimes A_{0}, \\
\tilde{C}_{0} & =I_{N} \otimes C_{0}, \\
\tilde{H}^{\mathrm{p}} & =L^{\mathrm{p}} \otimes I_{q}, \\
\tilde{H}^{\mathrm{c}} & =\operatorname{diag}\left\{\frac{1}{\sum_{j \in N_{i}^{\mathrm{c}}} g_{i j}^{\mathrm{c}}+g_{i 0}}\right\}\left(L^{\mathrm{c}}+G_{0}\right) \otimes I_{q},
\end{aligned}
$$

and substituting the protocol (6) into MAS (1) and (2), we can write the closed-loop dynamics of the overall network in the following compact form:

$$
\left\{\begin{array}{l}
\dot{x}_{l}=A_{l} x_{l}+B_{l} \tilde{x}_{0} \\
\dot{\tilde{x}}_{0}=\tilde{A}_{0} \tilde{x}_{0} \\
e=C_{l} x_{l}-\tilde{C}_{0} \tilde{x}_{0}
\end{array}\right.
$$

where

$$
\begin{aligned}
A_{l} & =\left[\begin{array}{cc}
\tilde{A}+\tilde{B} \tilde{K}_{x}-\tilde{D} \tilde{H}^{\mathrm{p}} \tilde{C} & \tilde{B} \tilde{K}_{z} \\
\tilde{S}_{2} \tilde{H}^{\mathrm{c}} \tilde{C} & \tilde{S}_{1}
\end{array}\right], \\
B_{l} & =\left[\begin{array}{c}
0 \\
-\tilde{S}_{2} \tilde{H}^{\mathrm{c}} \tilde{C}_{0}
\end{array}\right], \\
C_{l} & =\left[\begin{array}{ll}
\tilde{C} & 0
\end{array}\right] .
\end{aligned}
$$

It can be seen from (9) that the problem of output consensus will be solved if the global output error $e(t)=$ $C_{l} x_{l}(t)-\tilde{C}_{0} \tilde{x}_{0}(t) \rightarrow 0$ as $t \rightarrow \infty$. The following theorem gives a global sufficient condition on the system matrix $A_{l}$ for the problem.

Theorem 1. Consider the heterogeneous MAS (1) and (2) and let Assumptions 2-3 be satisfied. Then, the output consensus problem is solvable by the state-feedback protocol (6) if the system matrix $A_{l}$ in (10) is Hurwitz.

Proof 1. Assume that $A_{l}$ in (10) is Hurwitz. According to the output regulation theory (Huang [36]), the global output error $e(t)=C_{l} x_{l}-\tilde{C}_{0} \tilde{x}_{0}$ in (9) will converge to zero if the linear matrix equation:

$$
\left\{\begin{array}{l}
X_{l} \tilde{A}_{0}=A_{l} X_{l}+B_{l} \\
0=C_{l} X_{l}-\tilde{C}_{0}
\end{array}\right.
$$

has a unique solution $X_{l}$.

It is now required to show that under the hypotheses of this theorem, the unique solution $X_{l}$ to (11) indeed exists. Recall that since $A_{l}$ is exponentially stable and $\tilde{A}_{0}$ is completely unstable under Assumption 2, $\sigma\left(A_{l}\right) \cap \sigma\left(\tilde{A}_{0}\right)=$ $\varnothing$. Then, the first equation of (11) which is a Sylvester equation has a unique solution $X_{l}$. By decomposing $X_{l}$ as $X_{l}=$ $\left[X_{l 1}^{\mathrm{T}}, X_{l 2}^{\mathrm{T}}\right]^{\mathrm{T}}$, the first equation of (11) can be expanded as

$$
\left\{\begin{array}{l}
X_{l 1} \tilde{A}_{0}=\left[\tilde{A}-\tilde{D} \tilde{H}^{\mathrm{p}} \tilde{C}+\tilde{B} \tilde{K}_{x}\right] X_{l 1}+\tilde{B} \tilde{K}_{z} X_{l 2} \\
X_{l 2} \tilde{A}_{0}=\tilde{S}_{1} X_{l 2}+\tilde{S}_{2} \tilde{H}^{\mathrm{c}}\left(\tilde{C} X_{l 1}-\tilde{C}_{0}\right)
\end{array}\right.
$$

Since the pair $\left(S_{1 i}, S_{2 i}\right)$ incorporates a $q$-copy internal model of $A_{0}$, the pair $\left(\tilde{S}_{1}, \tilde{S}_{2}\right)$ also incorporates a $q$-copy internal model of $\tilde{A}_{0}$. So the solution $X_{l}=\left[X_{l 1}^{\mathrm{T}}, X_{l 2}^{\mathrm{T}}\right]^{\mathrm{T}}$ can guarantee that $0=C_{l} X_{l}-\tilde{C}_{0}$ with the help of Assumption 3 . Therefore, $X_{l}$ satisfies both equations of (11). This completes the proof.

3.2. Local Design for Control Gains. Based on Theorem 1, what remains is to design gain matrices $K_{x_{i}}$ and $K_{z_{i}}$ to make $A_{l}$ in (10) Hurwitz, which is equivalent to stabilizing the following linear systems coupled through graphs $\mathscr{G}^{\mathrm{p}}$ and $\mathscr{G}^{\mathrm{c}}$ :

$$
\left\{\begin{array}{l}
\bar{x}_{i}=\bar{A}_{i} \bar{x}_{i}-\left[\begin{array}{c}
D_{i} \\
0
\end{array}\right] \sum_{j \in N_{i}^{\mathrm{p}}} l_{i j}^{\mathrm{p}} \bar{y}_{j}-\left[\begin{array}{c}
0 \\
S_{2 i}
\end{array}\right] \frac{1}{\sum_{j \in N_{i}^{\mathrm{c}}} g_{i j}^{\mathrm{c}}+g_{i 0}} \sum_{j \in N_{i}^{\mathrm{c}}} g_{i j}^{\mathrm{c}} \bar{y}_{j}, \\
\bar{y}_{i}=\bar{C}_{i} \bar{x}_{i}, \quad i \in \mathscr{V},
\end{array}\right.
$$

where $\bar{x}_{i}=\left[x_{i}^{\mathrm{T}}, z_{i}^{\mathrm{T}}\right]^{\mathrm{T}}$ and

$$
\begin{aligned}
\bar{A}_{i} & =\left[\begin{array}{cc}
A_{i}+B_{i} K_{x_{i}} & B_{i} K_{z_{i}} \\
S_{2 i} C_{i} & S_{1 i}
\end{array}\right], \\
\bar{C}_{i} & =\left[\begin{array}{ll}
C_{i} & 0
\end{array}\right] .
\end{aligned}
$$


By letting $\bar{x}=\left[\bar{x}_{1}^{\mathrm{T}}, \ldots, \bar{x}_{N}^{\mathrm{T}}\right]^{\mathrm{T}}, \bar{y}=\left[\bar{y}_{1}^{\mathrm{T}}, \ldots, \bar{y}_{N}^{\mathrm{T}}\right]^{\mathrm{T}}$, and

$$
\begin{aligned}
& \bar{B}_{i}=\left[-\left[\begin{array}{c}
D_{i} \\
0
\end{array}\right],-\left[\begin{array}{c}
0 \\
S_{2 i}
\end{array}\right]\right], \\
& \bar{\Theta}=\operatorname{diag}\left\{\bar{\Theta}_{i}\right\}(\Theta=A, B, C), \\
& \Psi=L^{\mathrm{p}} \otimes\left[\begin{array}{c}
I_{q} \\
0
\end{array}\right]+\bar{G}^{\mathrm{c}} \otimes\left[\begin{array}{c}
0 \\
I_{q}
\end{array}\right], \\
& \bar{G}^{\mathrm{c}}=\operatorname{diag}\left\{\frac{1}{\sum_{j \in N_{i}^{c}} g_{i j}^{\mathrm{c}}+g_{i 0}}\right\} G^{\mathrm{c}},
\end{aligned}
$$

we can express the global form of system (13) as

$$
\left\{\begin{array}{l}
\bar{x}=\bar{A} \bar{x}+\bar{B} \Psi \bar{y} \\
\bar{y}=\bar{C} \bar{x}
\end{array}\right.
$$

Lemma 1. Suppose the transfer function $T_{i}(s)=\bar{C}_{i}$ $\left(s I_{n}-\bar{A}_{i}\right)^{-1} \bar{B}_{i}(\forall i \in \mathscr{V})$ is stable. Then, $A_{l}$ in (10) is Hurwitz if

$$
\max _{i \in \mathscr{V}}\left\|T_{i}(s)\right\|_{\infty}<\frac{1}{\bar{\sigma}(\Psi)},
$$

where $\left\|T_{i}(s)\right\|_{\infty}$ denotes the $H_{\infty}$ norm defined by $\left\|T_{i}(s)\right\|_{\infty}$ $=\sup _{\omega \in R} \bar{\sigma}\left(T_{i}(j \omega)\right)$.

Proof 2. Notice that stability of matrix $A_{l}$ in (10) is equivalent to stability of system (16). According to the small-gain theorem (Khalil [37]), system (16) is stable if

$$
\|T(s)\|_{\infty}\|\Psi\|_{\infty}<1
$$

where $T(s)=\operatorname{diag}\left\{T_{1}(s), \ldots, T_{N}(s)\right\}$.

It follows from

$$
\begin{aligned}
\|T(s)\|_{\infty}\|\Psi\|_{\infty} & =\left\|\operatorname{diag}\left\{T_{1}(s), \ldots, T_{N}(s)\right\}\right\|_{\infty} \bar{\sigma}(\Psi) \\
& \leq \max _{i \in \mathcal{N}}\left\|T_{i}(s)\right\|_{\infty} \bar{\sigma}(\Psi)
\end{aligned}
$$

that (17) is a sufficient condition for (18). This completes the proof.

Remark 2. Very recently, the cooperative output regulation problem was studied for heterogeneous MASs in a similar $H_{\infty}$ control framework [23], where both state feedback and dynamic output feedback were considered. Interestingly, Li. et al. [23] proposed a less conservative small-gain-type solvability condition than the ones in $[21,22]$. The main characteristic of the small-gain-type condition of [23] is that the communication graph is not separated from the $H_{\infty}$-type index of all agents, which is directly used for the graph weight optimization and $H_{\infty}$-control-based protocol gain design. Particularly, the square structure of the communication graph-related matrix in [23] allows that the solvability condition can be preexplicitly ensured for three special cases of the graph, the agent dynamcis and the exo-system dynamics. However, it can be seen from our graph-related matrix $\Psi$ in
(15) that the introduction of the physical graph $\mathscr{G}^{\mathrm{p}}$ not only destroys the square structure of $\Psi$ but also may lead to $\bar{\sigma}(\Psi)>1$. Therefore, the developed graph weight optimization method and the feasibility discussion for special MASs in [23] are not applicable to our considered MAS (1) and (2) with both physical graph $\mathscr{G}^{\mathrm{p}}$ and communication graph $\mathscr{G}^{\mathrm{c}}$.

By Theorem 1 and Lemma 1, the output consensus is linked to the $H_{\infty}$ control theory. In order to obtain the state-feedback protocol (6) that makes MAS (1) and (2) achieves output consensus, it suffices to find gains $K_{x_{i}}$ and $K_{z_{i}}$ such that a state-feedback controller internally stabilizes the linear system $T_{i}(s)$ with disturbance attenuation bound 1 $/ \bar{\sigma}(\Psi)$. For this purpose, we should consider the linear system:

$$
\left\{\begin{array}{l}
\dot{\hat{x}}_{i}=\widehat{A}_{i} \widehat{x}_{i}+\widehat{B}_{i} \widehat{u}_{i}+\bar{B}_{i} \widehat{w}_{i}, \\
\widehat{z}_{i}=\bar{C}_{i} \widehat{x}_{i},
\end{array}\right.
$$

under the state-feedback control:

$$
\widehat{u}_{i}=\left[\begin{array}{ll}
K_{x_{i}} & K_{z_{i}}
\end{array}\right] \widehat{x}_{i}
$$

Here, $\widehat{x}_{i} \in \mathbb{R}^{n_{i}+s_{i}}$ is the state, $\widehat{u}_{i} \in \mathbb{R}^{m_{i}}$ is the control input, $\widehat{w}_{i} \in \mathbb{R}^{2 q}$ is the disturbance input, $\widehat{z}_{i} \in \mathbb{R}^{q}$ is the controlled output, $\bar{C}_{i}$ and $\bar{B}_{i}$ are as in (14) and (15), and

$$
\begin{aligned}
& \widehat{A}_{i}=\left[\begin{array}{cc}
A_{i} & 0 \\
S_{2 i} C_{i} & S_{1 i}
\end{array}\right], \\
& \widehat{B}_{i}=\left[\begin{array}{c}
B_{i} \\
0
\end{array}\right] .
\end{aligned}
$$

The following result explains how to obtain control gains $K_{x_{i}}$ and $K_{z_{i}}$ such that (21) solves the $H_{\infty}$ problem of (20) with a prescribed disturbance attenuation constraint $\gamma>0$.

Lemma 2. Let $\gamma>0$ be a prescribed level of disturbance attenuation and $\delta_{i}>0$ be a given constant. Then, the linear system (20) is stabilizable with an $H_{\infty}$-norm bound $\gamma$ via the statefeedback controller (21) with gains given by

$$
\left[\begin{array}{ll}
K_{x_{i}} & K_{z_{i}}
\end{array}\right]=-\widehat{B}_{i}^{\mathrm{T}} \widehat{P}_{i}
$$

if there exists a positive definite solution $\widehat{P}_{i}>0$ for the Riccati equation:

$$
\widehat{A}_{i}^{\mathrm{T}} \widehat{P}_{i}+\widehat{P}_{i} \widehat{A}_{i}+\widehat{P}_{i}\left(\frac{1}{\gamma^{2}} \bar{B}_{i} \bar{B}_{i}^{\mathrm{T}}-2 \widehat{B}_{i} \widehat{B}_{i}^{\mathrm{T}}\right) \widehat{P}_{i}+\bar{C}_{i}^{\mathrm{T}} \bar{C}_{i}+\delta_{i} I=0 .
$$

Proof 3. When we apply the control (21) to the linear system (20), the closed-loop system from $\widehat{w}_{i}$ to $\widehat{z}_{i}$ is given by

$$
\left\{\begin{array}{l}
\dot{\hat{x}}_{i}=\bar{A}_{i} \widehat{x}_{i}+\bar{B}_{i} \widehat{w}_{i}, \\
\widehat{z}_{i}=\bar{C}_{i} \widehat{x}_{i},
\end{array}\right.
$$


Step 1 . Choose the pair $\left(S_{1 i}, S_{2 i}\right)$ to contain a $q$-copy internal model of $A_{0}$.

Step 2. For the given physical coupling graph $\mathscr{G}^{\mathrm{p}}$ and communication graph $\mathscr{G}^{\mathrm{c}}$, compute $\bar{\sigma}(\Psi)$ for $\Psi$ in (15).

Step 3. Choose the disturbance attenuation constraint $\gamma$ such that $0<\gamma \leq 1 / \bar{\sigma}(\Psi)$ and $\delta_{i}>0$.

Step 4. Solve the Riccati equation (24) to get gain matrices $K_{x_{i}}$ and $K_{z_{i}}$ according to (23).

Algorithm 1

with $\bar{A}_{i}$ in (14) expressed as

$$
\bar{A}_{i}=\widehat{A}_{i}-\widehat{B}_{i} \widehat{B}_{i}^{\mathrm{T}} \widehat{P}_{i}
$$

We first prove the asymptotic stability of the closedloop system (25) without external disturbance (i.e., $\widehat{w}_{i}=0$ ), by considering the Lyapunov function (Xie and de Souza Carlos [38])

$$
V(t)=\widehat{x}_{i}^{\mathrm{T}} \widehat{P}_{i} \widehat{x}_{i}
$$

where $\widehat{P}_{i}>0$ is a solution of (24).

Differentiating $V(t)$ along the trajectory of the closedloop system (25) yields

$$
\begin{aligned}
\dot{V}(t) & =\widehat{x}_{i}^{\mathrm{T}}\left(\bar{A}_{i}^{\mathrm{T}} \widehat{P}_{i}+\widehat{P}_{i} \bar{A}_{i}\right) \widehat{x}_{i} \\
& =\widehat{x}_{i}^{\mathrm{T}}\left(\widehat{A}_{i}^{\mathrm{T}} \widehat{P}_{i}+\widehat{P}_{i} \widehat{A}_{i}-2 \widehat{P}_{i} \widehat{B}_{i} \widehat{B}_{i}^{\mathrm{T}} \widehat{P}_{i}\right) \widehat{x}_{i} \\
& =-\widehat{x}_{i}^{\mathrm{T}}\left(\frac{1}{\gamma_{i}^{2}} \widehat{P}_{i} \bar{B}_{i} \bar{B}_{i}^{\mathrm{T}} \widehat{P}_{i}+\bar{C}_{i}^{\mathrm{T}} \bar{C}_{i}+\delta_{i} I\right) \widehat{x}_{i}<0,
\end{aligned}
$$

then the closed-loop system (25) is asymptotically stable with $\widehat{w}_{i}=0$.

Next, we establish the upper bound $\gamma\left\|\widehat{w}_{i}\right\|_{2}$ for the $L_{2}[0, \infty)$ norm of $\widehat{w}_{i}$ by introducing

$$
J=\int_{0}^{\infty}\left[\widehat{z}_{i}^{\mathrm{T}} \widehat{z}_{i}-\gamma^{2} \widehat{w}_{i}^{\mathrm{T}} \widehat{w}_{i}\right] d t
$$

Assuming $\widehat{x}_{i}(0)=0$, we have that for any nonzero $\widehat{w}_{i}$ $\in L_{2}[0, \infty)$ :

$$
\begin{aligned}
J= & \int_{0}^{\infty}\left[\widehat{z}_{i}^{\mathrm{T}} \widehat{z}_{i}-\gamma^{2} \widehat{w}_{i}^{\mathrm{T}} \widehat{w}_{i}\right] d t \\
= & \int_{0}^{\infty}\left[\widehat{z}_{i}^{\mathrm{T}} \widehat{z}_{i}-\gamma^{2} \widehat{w}_{i}^{\mathrm{T}} \widehat{w}_{i}+\frac{d}{d t}\left(\widehat{x}_{i}^{\mathrm{T}} \widehat{P}_{i} \widehat{x}_{i}\right)\right] d t-\widehat{x}_{i}^{\mathrm{T}}(\infty) \widehat{P}_{i} \widehat{x}_{i}(\infty) \\
= & \int_{0}^{\infty} \widehat{x}_{i}^{\mathrm{T}}\left[\widehat{A}_{i}^{\mathrm{T}} \widehat{P}_{i}+\widehat{P}_{i} \widehat{A}_{i}+\widehat{P}_{i}\left(\frac{1}{\gamma^{2}} \bar{B}_{i} \bar{B}_{i}^{\mathrm{T}}-2 \widehat{B}_{i} \widehat{B}_{i}^{\mathrm{T}}\right) \widehat{P}_{i}+\bar{C}_{i}^{\mathrm{T}} \bar{C}_{i}\right] \widehat{x}_{i} d t \\
& -\int_{0}^{\infty} \gamma^{2}\left[\widehat{w}_{i}-\frac{1}{\gamma^{2}} \bar{B}_{i}^{\mathrm{T}} \widehat{P}_{i} \widehat{x}_{i}\right]^{\mathrm{T}}\left[\widehat{w}_{i}-\frac{1}{\gamma^{2}} \bar{B}_{i}^{\mathrm{T}} \widehat{P}_{i} \widehat{x}_{i}\right] d t \\
& -\widehat{x}_{i}^{\mathrm{T}}(\infty) \widehat{P}_{i} \widehat{x}_{i}(\infty) .
\end{aligned}
$$

Considering (24), one can show that $J<0$, i.e., $\left\|\widehat{z}_{i}\right\|_{2}$ $\leq \gamma\left\|\widehat{w}_{i}\right\|_{2}$ for any nonzero $\widehat{w}_{i} \in L_{2}[0, \infty)$. This ends the proof.
Based on Theorem 1 and Lemmas 1-2, the following theorem yields a state-feedback protocol for output consensus of heterogeneous networks.

Theorem 2. For any given $0<\gamma \leq 1 / \bar{\sigma}(\Psi)$, consider the heterogeneous MAS (1) and (2) under Assumptions 1-4. The output consensus problem is solved by the state-feedback protocol (6) if we design control gains $K_{x_{i}}$ and $K_{z_{i}}$ as in (23) where $\widehat{P}_{i}>0$ is the solution of the Riccati equation (24).

Remark 3. From Theorem 2, the state-feedback protocol (6) can be designed along the following steps (Algorithm 1).

\section{The Case of Output-Feedback Design}

We now deal with the output-feedback design for achieving output consensus of system (1) and (2), which is of the form:

$$
\left\{\begin{array}{l}
u_{i}=K_{y_{i}} y_{i}+K_{z_{i}} z_{i}, \\
\dot{z}_{i}=S_{1 i} z_{i}+S_{2 i} e_{i v}
\end{array}\right.
$$

where $z_{i} \in \mathbb{R}^{s_{i}}$ represents the internal controller state and $K_{y_{i}}$ and $K_{z_{i}}$ are the output-feedback gain and feedforward gain to be determined, respectively. $S_{1 i}$ and $S_{2 i}$ are constant matrices such that the pair $\left(S_{1 i}, S_{2 i}\right)$ contains a $q$-copy internal model as defined in Definition 2 of $A_{0}$.

Interconnecting the MAS (1) and (2) using the outputfeedback protocol (31), the closed-loop system can be still obtained as (9) but with only $A_{l}$ in (10) redefined by

$$
A_{l}=\left[\begin{array}{cc}
\tilde{A}+\tilde{B} \tilde{K}_{y} \tilde{C}-\tilde{D} \tilde{H}^{p} \tilde{C} & \tilde{B} \tilde{K}_{z} \\
\tilde{S}_{2} \tilde{H}^{c} \tilde{C} & \tilde{S}_{1}
\end{array}\right] .
$$

Therefore, Theorem 1 holds to solve the output consensus problem by the output-feedback protocol (31) with the corresponding system matrix $A_{l}$ in (32). Similarly, Lemma 1 holds with $\bar{A}_{i}$ in (14) redefined by

$$
\bar{A}_{i}=\left[\begin{array}{cc}
A_{i}+B_{i} K_{y_{i}} C_{i} & B_{i} K_{z_{i}} \\
S_{2 i} C_{i} & S_{1 i}
\end{array}\right] .
$$

As in Section 3 for the case of state-feedback design, we now consider the linear system:

$$
\left\{\begin{array}{l}
\dot{\hat{x}}_{i}=\widehat{A}_{i} \widehat{x}_{i}+\widehat{B}_{i} \widehat{u}_{i}+\bar{B}_{i} \widehat{w}_{i}, \\
\widehat{y}_{i}=\widehat{C}_{i} \widehat{x}_{i}, \\
\widehat{z}_{i}=\bar{C}_{i} \widehat{x}_{i},
\end{array}\right.
$$


Step 1 . Choose the pair $\left(S_{1}, S_{2 i}\right)$ to contain a $q$-copy internal model of $A_{0}$.

Step 2. For the given physical coupling graph $\mathscr{G}^{\mathrm{p}}$ and communication graph $\mathscr{G}^{\mathrm{c}}$, compute $\bar{\sigma}(\Psi)$ for $\Psi$ in (15).

Step 3. Choose the disturbance attenuation constraint $\gamma$ such that $0<\gamma \leq 1 / \bar{\sigma}(\Psi)$ and $\delta_{i}>0$.

Step 4. Set $n=0$ and $L_{i}(0)=0$.

Step 5. Find $\widehat{P}_{i}(n)$ through solving

$$
\widehat{A}_{i}^{\mathrm{T}} \widehat{P}_{i}(n)+\widehat{P}_{i}(n) \widehat{A}_{i}+\widehat{P}_{i}(n)\left(\frac{1}{\gamma^{2}} \bar{B}_{i} \bar{B}_{i}^{\mathrm{T}}-2 \widehat{B}_{i} \widehat{B}_{i}^{\mathrm{T}}\right) \widehat{P}_{i}(n)+\bar{C}_{i}^{\mathrm{T}} \bar{C}_{i}-L_{i}^{\mathrm{T}}(n) \widehat{B}_{i}^{\mathrm{T}} \widehat{P}_{i}(n)-\widehat{P}_{i}(n) \widehat{B}_{i} L_{i}(n)+\delta_{i} I=0 .
$$

Update the control gain $\left[\begin{array}{ll}K_{y_{i}} & K_{z_{i}}\end{array}\right]$ and the matrix $L_{i}$ using

$$
\left[\begin{array}{ll}
K_{y_{i}}(n+1) & K_{z_{i}}(n+1)
\end{array}\right]=-\left(\widehat{B}_{i}^{\mathrm{T}} \widehat{P}_{i}(n)+L_{i}(n)\right) \widehat{C}_{i}^{\mathrm{T}}\left(\widehat{C}_{i} \widehat{C}_{i}^{\mathrm{T}}\right)^{-1}, L_{i}(n+1)=-\left[K_{y_{i}}(n+1) \quad K_{z_{i}}(n+1)\right] \widehat{C}_{i}-\widehat{B}_{i}^{\mathrm{T}} P_{i}(n) .
$$

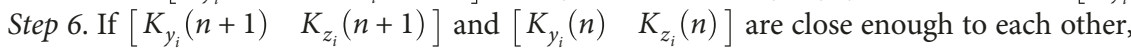
stop and set $\left[\begin{array}{ll}K_{y_{i}} & K_{z_{i}}\end{array}\right]=\left[\begin{array}{ll}K_{y_{i}}(n+1) & K_{z_{i}}(n+1)\end{array}\right]$. Otherwise, set $n=n+1$ and go to Step 5 .

Algorithm 2

under the output-feedback control:

$$
\widehat{u}_{i}=\left[\begin{array}{ll}
K_{y_{i}} & K_{z_{i}}
\end{array}\right] \widehat{y}_{i}
$$

Here, $\widehat{x}_{i} \in \mathbb{R}^{n_{i}+s_{i}}$ is the state, $\widehat{u}_{i} \in \mathbb{R}^{m_{i}}$ is the control input, $\widehat{w}_{i} \in \mathbb{R}^{2 q}$ is the disturbance input, $\widehat{y}_{i} \in \mathbb{R}^{q+s_{i}}$ is the measured output, $\widehat{z}_{i} \in \mathbb{R}^{q}$ is the controlled output, $\bar{C}_{i}$ and $\bar{B}_{i}$ are as in (14) and (15), $\widehat{A}_{i}$ and $\widehat{B}_{i}$ are as in (22), and

$$
\widehat{C}_{i}=\left[\begin{array}{cc}
C_{i} & 0 \\
0 & I
\end{array}\right] .
$$

Motivated by the static output-feedback design for the single agents [39], we establish the following Lemma on how to compute output-feedback control for the $H_{\infty}$ control problem of linear systems.

Lemma 3. Let $\gamma>0$ be a prescribed level of disturbance attenuation and $\delta_{i}>0$ be a given constant. The linear system (34) is stabilizable with an $H_{\infty}$-norm bound $\gamma$ via the outputfeedback controller (35) if there exist matrices $K_{y_{i}}, K_{z_{i}}$, and $L_{i}$ such that

$$
\left[\begin{array}{ll}
K_{y_{i}} & K_{z_{i}}
\end{array}\right] \widehat{C}_{i}=-\left(\widehat{B}_{i}^{\mathrm{T}} \widehat{P}_{i}+L_{i}\right)
$$

where $\widehat{P}_{i}>0$ is a solution of the Riccati equation:

$$
\begin{gathered}
\widehat{A}_{i}^{\mathrm{T}} \widehat{P}_{i}+\widehat{P}_{i} \widehat{A}_{i}+\widehat{P}_{i}\left(\frac{1}{\gamma^{2}} \bar{B}_{i} \bar{B}_{i}^{\mathrm{T}}-2 \widehat{B}_{i} \widehat{B}_{i}^{\mathrm{T}}\right) \widehat{P}_{i} \\
+\bar{C}_{i}^{\mathrm{T}} \bar{C}_{i}-L_{i}^{\mathrm{T}} \widehat{B}_{i}^{\mathrm{T}} \widehat{P}_{i}-\widehat{P}_{i} \widehat{B}_{i} L_{i}+\delta_{i} I=0 .
\end{gathered}
$$

Proof 4. The proof is similar to Lemma 2 and is thus omitted here.

Based on Theorem 1 and Lemmas 1 and 3, the main result on output consensus using the output-feedback design is established below.
Theorem 3. For any given $0<\gamma<1 / \bar{\sigma}(\Psi)$, consider the heterogeneous MAS (1) and (2) under Assumptions 1-4. The output consensus problem is solved by the output-feedback protocol (31) if we design control gains $K_{y_{i}}$ and $K_{z_{i}}$ as in (37) where $\widehat{P}_{i}>0$ is the solution of the Riccati equation (38).

Remark 4. From Theorem 3, the output-feedback protocol (31) should be designed by solving the coupled matrix (37) and (38). These equations can be efficiently solved by the following steps (Algorithm 2) without requiring the initial stabilizing gains [39].

\section{Simulation Results}

Consider a group of one leader and three followers with the communication network topology $\mathscr{G}^{\mathrm{c}}$ and the physical network topology $\mathscr{G}^{\mathrm{P}}$ as shown in Figure 1. The corresponding Laplacian matrices $L^{\mathrm{c}}$ and $L^{\mathrm{p}}$ are given by

$$
\begin{aligned}
L^{\mathrm{c}} & =\left(\begin{array}{ccc}
0.1 & 0 & -0.1 \\
0 & 0 & 0 \\
-0.02 & -0.04 & 0.06
\end{array}\right), \\
L^{\mathrm{p}} & =\left(\begin{array}{ccc}
0.06 & -0.06 & 0 \\
0 & 0.02 & -0.02 \\
0 & 0 & 0
\end{array}\right),
\end{aligned}
$$

with the leader adjacency matrix $G_{0}=\operatorname{diag}\{0,1,1\}$.

The dynamics of the leader and each follower is described as below.

$$
\left\{\begin{array}{l}
\dot{x}_{0}=\left[\begin{array}{cc}
1 & 2 \\
-3 & -1
\end{array}\right] x_{0} \\
y_{0}=\left[\begin{array}{ll}
1 & 0
\end{array}\right] x_{0},
\end{array}\right.
$$




$$
\begin{aligned}
& \left\{\begin{array}{l}
\dot{x}_{1}=x_{1}+u_{1}+\sum_{j \in N_{1}^{\mathrm{p}}} g_{1 j}^{\mathrm{p}}\left(y_{j}-y_{1}\right), \\
y_{1}=x_{1}
\end{array}\right. \\
& \left\{\begin{array}{l}
\dot{x}_{2}=\left[\begin{array}{ll}
0 & 1 \\
1 & 0
\end{array}\right] x_{2}+\left[\begin{array}{l}
0 \\
1
\end{array}\right] u_{2}+\left[\begin{array}{l}
1 \\
1
\end{array}\right] \sum_{j \in N_{2}^{\mathrm{p}}} g_{2 j}^{\mathrm{p}}\left(y_{j}-y_{2}\right), \\
y_{2}=\left[\begin{array}{ll}
1 & 0
\end{array}\right] x_{2}
\end{array}\right. \\
& \left\{\begin{array}{l}
\dot{x}_{3}=\left[\begin{array}{ccc}
0 & -2 & 0 \\
0 & 0 & -3 \\
0 & 0 & 1
\end{array}\right] x_{3}+\left[\begin{array}{ll}
2 & 0 \\
0 & 4 \\
1 & 0
\end{array}\right], \\
u_{3}+\left[\begin{array}{l}
0 \\
1 \\
1
\end{array}\right] \sum_{j \in N_{3}^{\mathrm{p}}} g_{3 j}^{\mathrm{p}}\left(y_{j}-y_{3}\right), \\
y_{3}=\left[\begin{array}{lll}
1 & 1 & 0
\end{array}\right] x_{3} .
\end{array}\right.
\end{aligned}
$$

$$
\begin{aligned}
& {\left[\begin{array}{ll}
K_{x_{1}} & K_{z_{1}}
\end{array}\right]=\left[\begin{array}{llll}
-1.8212 & 0.3001 & -0.6437
\end{array}\right],} \\
& {\left[\begin{array}{llll}
K_{x_{2}} & K_{z_{2}}
\end{array}\right]=\left[\begin{array}{lllll}
-2.4747 & -1.7395 & 2.1051 & 0.5652
\end{array}\right]} \\
& {\left[\begin{array}{lllll}
K_{x_{3}} & K_{z_{3}}
\end{array}\right]=\left[\begin{array}{lllll}
-0.5999 & -0.0816 & -1.0550 & -0.1554 & -0.4300 \\
1.0873 & -1.1951 & -2.5010 & -0.8602 & -0.3173
\end{array}\right] .}
\end{aligned}
$$

Thus, according to Theorem 2, MAS (40), (41), (42), and (43) has achieved the output consensus by using the statefeedback control (6) with control gains $K_{x_{i}}$ and $K_{z_{i}}$ in (45). The evolutions of the agent outputs and tracking errors are shown in Figures 2 and 3, respectively.

5.2. The Output-Feedback Case. In this case, we use the output-feedback protocol (31) with the output matrices $\widehat{C}_{i}$ in (34) as

$$
\begin{aligned}
& \widehat{C}_{1}=I_{3}, \\
& \widehat{C}_{2}=\left[\begin{array}{llll}
1 & 0 & 0 & 0 \\
0 & 0 & 1 & 0 \\
0 & 0 & 0 & 1
\end{array}\right], \\
& \widehat{C}_{3}=\left[\begin{array}{lllll}
1 & 1 & 0 & 0 & 0 \\
0 & 0 & 0 & 1 & 0 \\
0 & 0 & 0 & 0 & 1
\end{array}\right],
\end{aligned}
$$

and other parameters are kept the same as the statefeedback case.

Based on Algorithm 2 and setting $\gamma=8.0<1 / \bar{\sigma}(\Psi)$ and $\sigma_{1}=\sigma_{2}=\sigma_{3}=0.8$, we select the gain matrix $K_{y_{i}}$ and $K_{z_{i}}$ in (37) as follows:
It can be easily verified that Assumptions 1-4 are satisfied by designing the internal model $\left(S_{1 i}, S_{2 i}\right)$ for the $i$ th follower as

$$
\begin{aligned}
S_{1 i}= & {\left[\begin{array}{cc}
1 & 2 \\
-3 & -1
\end{array}\right], } \\
S_{2 i}= & {\left[\begin{array}{l}
0 \\
1
\end{array}\right], } \\
& i=1,2,3 .
\end{aligned}
$$

With the Laplacian matrices $L^{\mathrm{c}}$ and $L^{\mathrm{p}}$ in (39), we have $\bar{\sigma}(\Psi)=0.1022$ and $1 / \bar{\sigma}(\Psi)=9.7857$.

5.1. The State-Feedback Case. In this case, we use the state-feedback protocol (6). Based on Algorithm 1, we set $\gamma=6.0<1 / \bar{\sigma}(\Psi)$ and $\sigma_{1}=\sigma_{2}=\sigma_{3}=0.6$. Then, by solving the Riccati equation (24), the gain matrix $K_{x_{i}}$ and $K_{z_{i}}$ in (23) can be designed as

$$
\begin{aligned}
{\left[\begin{array}{ll}
K_{y_{1}} & K_{z_{1}}
\end{array}\right] } & =\left(\begin{array}{lll}
-1.8849 & 0.2938 & -0.7361
\end{array}\right), \\
L_{1} & =\left(\begin{array}{lll}
0 & 0 & 0
\end{array}\right), \\
{\left[\begin{array}{ll}
K_{y_{2}} & K_{z_{2}}
\end{array}\right] } & =\left(\begin{array}{llll}
-2.5862 & 2.3817 & 0.4205
\end{array}\right), \\
L_{2} & =\left(\begin{array}{llll}
0 & -2.7384 & 0 & 0
\end{array}\right), \\
{\left[\begin{array}{ll}
K_{y_{3}} & K_{z_{3}}
\end{array}\right] } & =\left(\begin{array}{llll}
-0.2330 & -0.5079 & -0.5760 \\
-0.0517 & -0.9173 & -0.2407
\end{array}\right), \\
L_{3} & =\left(\begin{array}{lllll}
-0.2747 & 0.2747 & -2.6205 & 0 & 0 \\
2.0712 & -2.0712 & -4.1245 & 0 & 0
\end{array}\right) .
\end{aligned}
$$

Thus, according to Theorem 3, MAS (40), (41), (42), and (43) has achieved output consensus by using the outputfeedback control (31) with control gains $K_{y_{i}}$ and $K_{z_{i}}$ in (47). The evolutions of the agent outputs and tracking errors are shown in Figures 4 and 5, respectively.

\section{Conclusion}

The output consensus problem has been solved for a group of heterogeneous linear agents whose dynamics are coupled by a physical coupling graph. Distributed control protocols relying on the relative output information have been proposed 


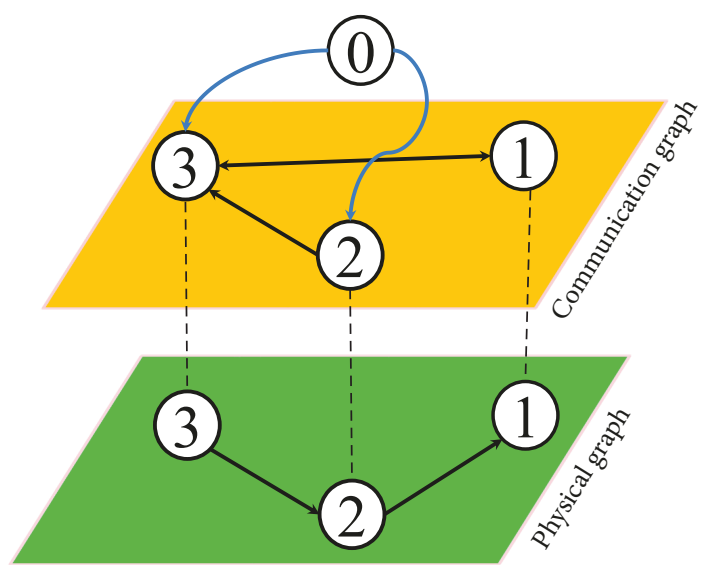

FIGURE 1: The physical graph $\mathscr{G}^{\mathrm{p}}$ and communication graph $\mathscr{G}^{\mathrm{c}}$ for simulation examples.

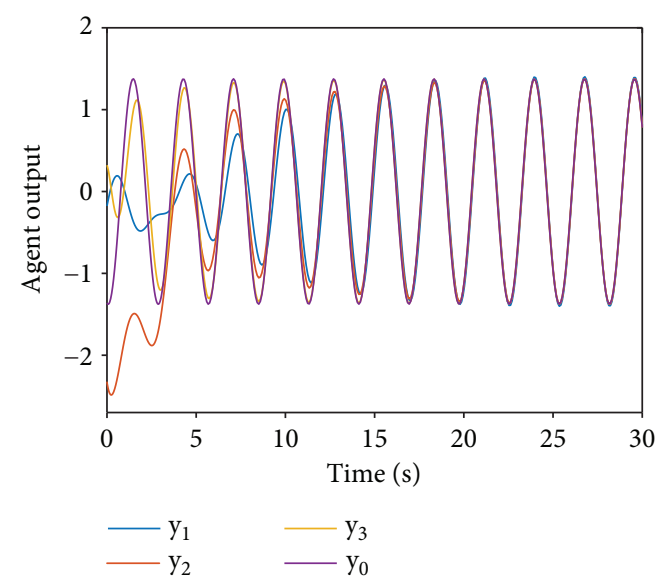

Figure 2: The follower output $y_{i}(i=1,2,3)$ and leader output $y_{0}$ by state-feedback control (6).

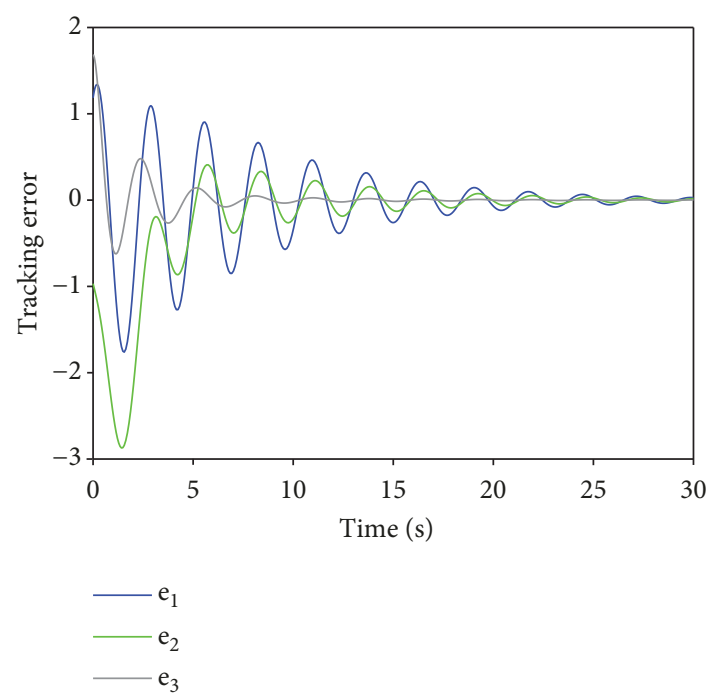

FIGURE 3: The tracking error $e_{i}=y_{i}-y_{0}(i=1,2,3)$ by state-feedback control (6).

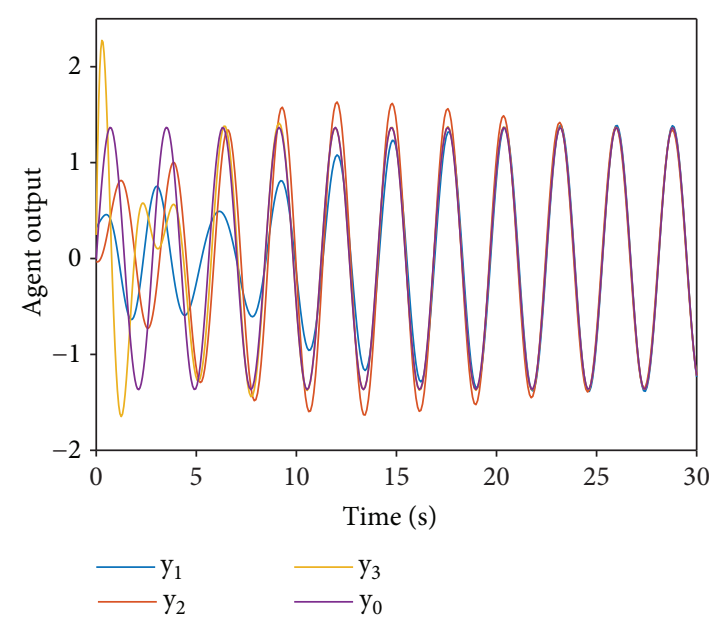

Figure 4: The follower output $y_{i}(i=1,2,3)$ and leader output $y_{0}$ by output-feedback control (31).

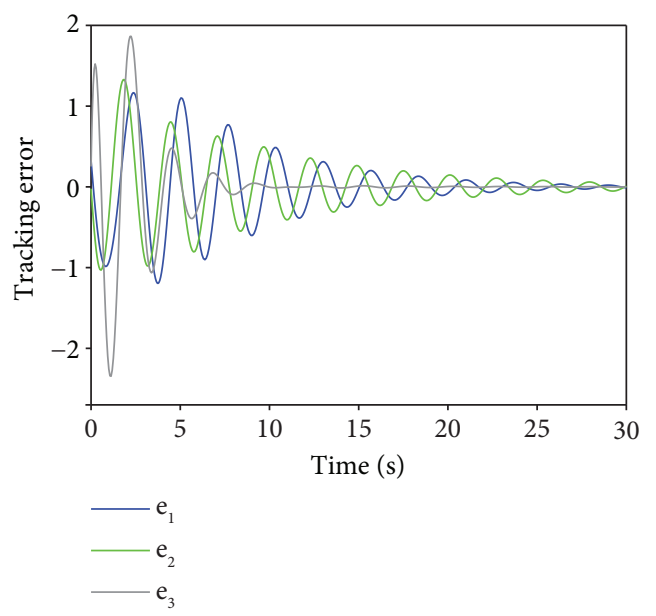

FIgURE 5: The tracking error $e_{i}=y_{i}-y_{0}(i=1,2,3)$ by outputfeedback control (31).

for both state-feedback and output-feedback cases. By bringing together output regulation theory and $H_{\infty}$ control technique, we have demonstrated that the developed protocols guarantee that the output of each follower synchronizes to that of the leader. The computational algorithms for computing control gains were provided by solving the algebraic Riccati equations. It should be noted that the recent work of Li. et al. [23] introduces an interesting topic of discussing the preexplicit issue for output consensus of special MASs. Unfortunately, until now, we have not established the similar results for MASs with multiplex interaction graphs (e.g., the communication graph $\mathscr{G}^{c}$ and the physical graph $\mathscr{G}^{\mathrm{p}}$ considered here), which leaves an open problem for our future study. Also, it is worth extending our results to solve the similar problem of uncertain MASs with complicated agent dynamics.

\section{Data Availability}

No data were used to support this study. 


\section{Conflicts of Interest}

The authors declare that they have no competing interests.

\section{Acknowledgments}

This work was jointly supported by the National Natural Science Foundation of China under Grant 11771102, the Characteristic Innovation Project of Education Department of Guangdong Province under Grant 2015KTSCX034, the Zhujiang New Star under Grant 201506010056, Guangdong Province Outstanding Young Teacher Training Plan under Grant YQ2015050, and by the Guangdong Natural Science Foundation under Grants 2014A030313438 and 2017A030313397.

\section{References}

[1] F. L. Lewis, H. Zhang, K. Hengster-Movric, and A. Das, Cooperative Control of Multi-Agent Systems: Optimal and Adaptive Design Approaches, Springer, 2014.

[2] W. Yu, G. Wen, G. Chen, and J. Cao, Distributed Cooperative Control of Multi-Agent Systems, Wiley, Newark, NJ, USA, 2016.

[3] J. Qin, Q. Ma, Y. Shi, and L. Wang, "Recent advances in consensus of multi-agent systems: a brief survey," IEEE Transactions on Industrial Electronics, vol. 64, no. 6, pp. 4972-4983, 2017.

[4] L. Ding, Q.-L. Han, X. Ge, and X.-M. Zhang, "An overview of recent advances in event-triggered consensus of multiagent systems," IEEE Transactions on Cybernetics, vol. 48, no. 4, pp. 1110-1123, 2018.

[5] R. Olfati-Saber, J. A. Fax, and R. M. Murray, "Consensus and cooperation in networked multi-agent systems," Proceedings of the IEEE, vol. 95, no. 1, pp. 215-233, 2007.

[6] Y. Cao, W. Yu, W. Ren, and G. Chen, "An overview of recent progress in the study of distributed multi-agent coordination," IEEE Transactions on Industrial Informatics, vol. 9, no. 1, pp. 427-438, 2013.

[7] L. Rong and H. Shen, "Distributed containment control of second-order multiagent systems with input delays under general protocols," Complexity, vol. 21, no. 6, p. 120, 2016.

[8] Y. Shang and Y. Ye, "Leader-follower fixed-time group consensus control of multiagent systems under directed topology," Complexity, vol. 2017, Article ID 3465076, 9 pages, 2017.

[9] S. E. Tuna, "Synchronizing linear systems via partial-state coupling," Automatica, vol. 44, no. 8, pp. 2179-2184, 2008.

[10] L. Scardovi and R. Sepulchre, "Synchronization in networks of identical linear systems," Automatica, vol. 45, no. 11, pp. 2557-2562, 2009.

[11] J. H. Seo, H. Shim, and J. Back, "Consensus of high-order linear systems using dynamic output feedback compensator: low gain approach," Automatica, vol. 45, no. 11, pp. 2659-2664, 2009.

[12] C.-Q. Ma and J.-F. Zhang, "Necessary and sufficient conditions for consensusability of linear multi-agent systems," IEEE Transactions on Automatic Control, vol. 55, no. 5, pp. 12631268, 2010.

[13] D. Fiore, G. Russo, and M. di Bernardo, "Exploiting nodes symmetries to control synchronization and consensus patterns in multiagent systems," IEEE Control Systems Letters, vol. 1, no. 2, pp. 364-369, 2017.

[14] H. Zhang and J. Chen, "Bipartite consensus of multi-agent systems over signed graphs: state feedback and output feedback control approaches," International Journal of Robust and Nonlinear Control, vol. 27, no. 1, pp. 3-14, 2017.

[15] X. Li, Y. C. Soh, and L. Xie, “Output-feedback protocols without controller interaction for consensus of homogeneous multi-agent systems: a unified robust control view," Automatica, vol. 81, pp. 37-45, 2017.

[16] P. Wieland, R. Sepulchre, and F. Allgöwer, “An internal model principle is necessary and sufficient for linear output synchronization," Automatica, vol. 47, no. 5, pp. 1068-1074, 2011.

[17] H. F. Grip, T. Yang, A. Saberi, and A. A. Stoorvogel, "Output synchronization for heterogeneous networks of nonintrospective agents," Automatica, vol. 48, no. 10, pp. 24442453, 2012.

[18] H. L. Trentelman, K. Takaba, and N. Monshizadeh, "Robust synchronization of uncertain linear multi-agent systems," IEEE Transactions on Automatic Control, vol. 58, no. 6, pp. 1511-1523, 2013.

[19] Y. Su and J. Huang, "Cooperative output regulation of linear multi-agent systems," IEEE Transactions on Automatic Control, vol. 57, no. 4, pp. 1062-1066, 2012.

[20] X. Wang, Y. Hong, J. Huang, and Z.-P. Jiang, “A distributed control approach to a robust output regulation problem for multi-agent linear systems," IEEE Transactions on Automatic Control, vol. 55, no. 12, pp. 2891-2895, 2010.

[21] C. Huang and X. Ye, "Cooperative output regulation of heterogeneous multi-agent systems: an $H_{\infty}$ criterion," IEEE Transactions on Automatic Control, vol. 59, no. 1, pp. 267273, 2014.

[22] F. A. Yaghmaie, F. L. Lewis, and R. Su, "Output regulation of linear heterogeneous multi-agent systems via output and state feedback," Automatica, vol. 67, pp. 157-164, 2016.

[23] X. Li, Y. C. Soh, L. Xie, and F. L. Lewis, "Cooperative output regulation of heterogeneous linear multi-agent networks via $H_{\infty}$ performance allocation," IEEE Transactions on Automatic Control, p. 1, 2018.

[24] W. Hu, L. Liu, and G. Feng, "Output consensus of heterogeneous linear multi-agent systems by distributed event-triggered/self-triggered strategy," IEEE Transactions on Cybernetics, vol. 47, no. 8, pp. 1914-1924, 2017.

[25] Y. Ma and J. Zhao, "Distributed integral-based event-triggered scheme for cooperative output regulation of switched multiagent systems," Information Sciences, vol. 457-458, pp. 208221, 2018.

[26] A. Abdessameud and A. Tayebi, "Distributed output regulation of heterogeneous linear multi-agent systems with communication constraints," Automatica, vol. 91, pp. 152-158, 2018.

[27] S. Zuo, Y. Song, F. L. Lewis, and A. Davoudi, “Output containment control of linear heterogeneous multi-agent systems using internal model principle," IEEE Transactions on Cybernetics, vol. 47, no. 8, pp. 2099-2109, 2017.

[28] A. Bidram, F. L. Lewis, and A. Davoudi, "Distributed control systems for small-scale power networks: using multiagent cooperative control theory," IEEE Control Systems, vol. 34, no. 6, pp. 56-77, 2014.

[29] J. Fink, A. Ribeiro, and V. Kumar, "Robust control for mobility and wireless communication in cyber-physical systems with 
application to robot teams," Proceedings of the IEEE, vol. 100, no. 1, pp. 164-178, 2012.

[30] X. F. Wang and G. Chen, "Pinning control of scale-free dynamical networks," Physica A: Statistical Mechanics and its Applications, vol. 310, no. 3-4, pp. 521-531, 2002.

[31] W. Yu, G. Chen, and J. Lü, "On pinning synchronization of complex dynamical networks," Automatica, vol. 45, no. 2, pp. 429-435, 2009.

[32] A. Bidram, A. Davoudi, F. L. Lewis, and J. M. Guerrero, "Distributed cooperative secondary control of microgrids using feedback linearization," IEEE Transactions on Power Systems, vol. 28, no. 3, pp. 3462-3470, 2013.

[33] T. Morstyn, B. Hredzak, G. D. Demetriades, and V. G. Agelidis, "Unified distributed control for DC microgrid operating modes," IEEE Transactions on Power Systems, vol. 31, no. 1, pp. 802-812, 2016.

[34] J. Wang, K. Chen, and F. L. Lewis, "Coordination of multiagent systems on interacting physical and communication topologies," Systems \& Control Letters, vol. 100, pp. 56-65, 2017.

[35] D. A. B. Lombana and M. di Bernardo, "Multiplex PI control for consensus in networks of heterogeneous linear agents," Automatica, vol. 67, pp. 310-320, 2016.

[36] J. Huang, Nonlinear Output Regulation: Theory and Applications, SIAM, 2004.

[37] H. K. Khalil, Nonlinear Systems, Prentice Hall, 3rd edition, 2002.

[38] L. Xie and E. de Souza Carlos, "Robust $H_{\infty}$ control for linear systems with norm-bounded time-varying uncertainty," IEEE Transactions on Automatic Control, vol. 37, no. 8, pp. 11881191, 1992.

[39] J. Gadewadikar, F. L. Lewis, and M. Abu-Khalaf, "Necessary and sufficient conditions for $H$ - $\infty$ static output-feedback control," Journal of Guidance, Control, and Dynamics, vol. 29, no. 4, pp. 915-920, 2006. 


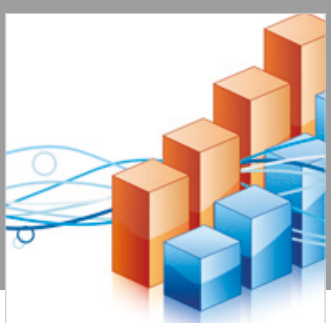

Advances in

Operations Research

\section{-n-m}
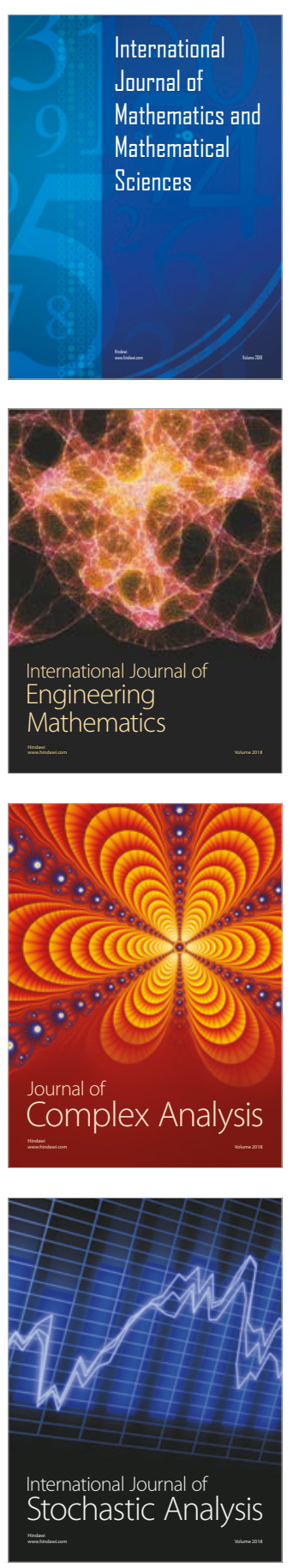
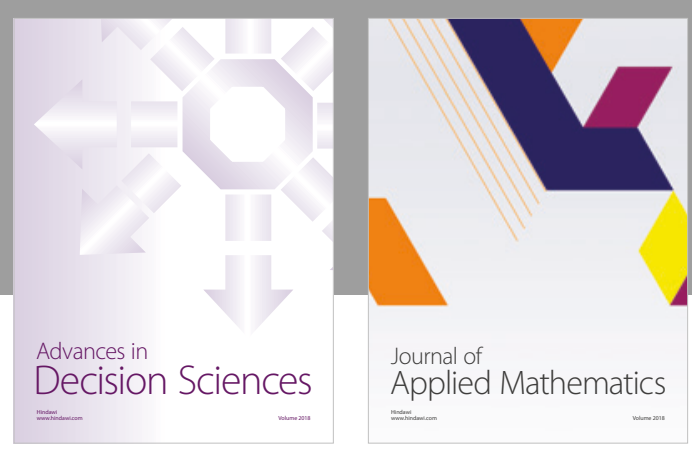

Journal of

Applied Mathematics
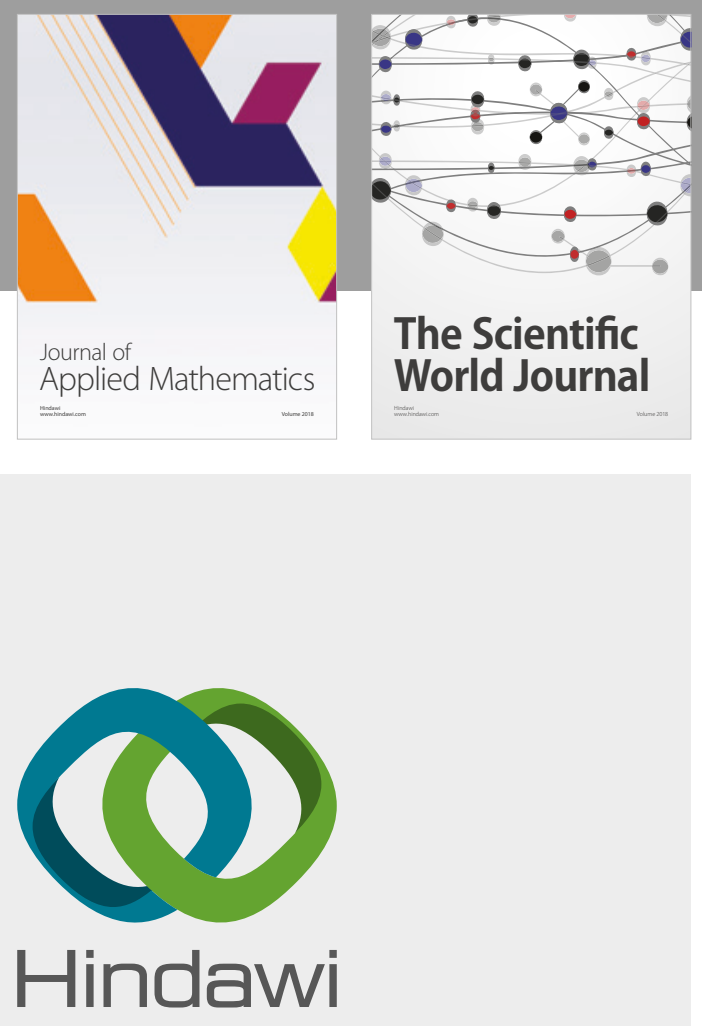

Submit your manuscripts at

www.hindawi.com

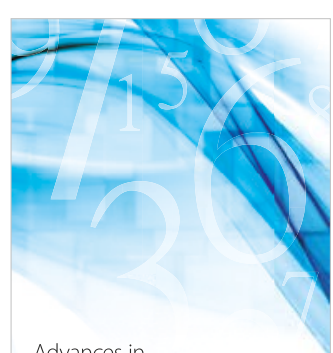

Advances in
Numerical Analysis
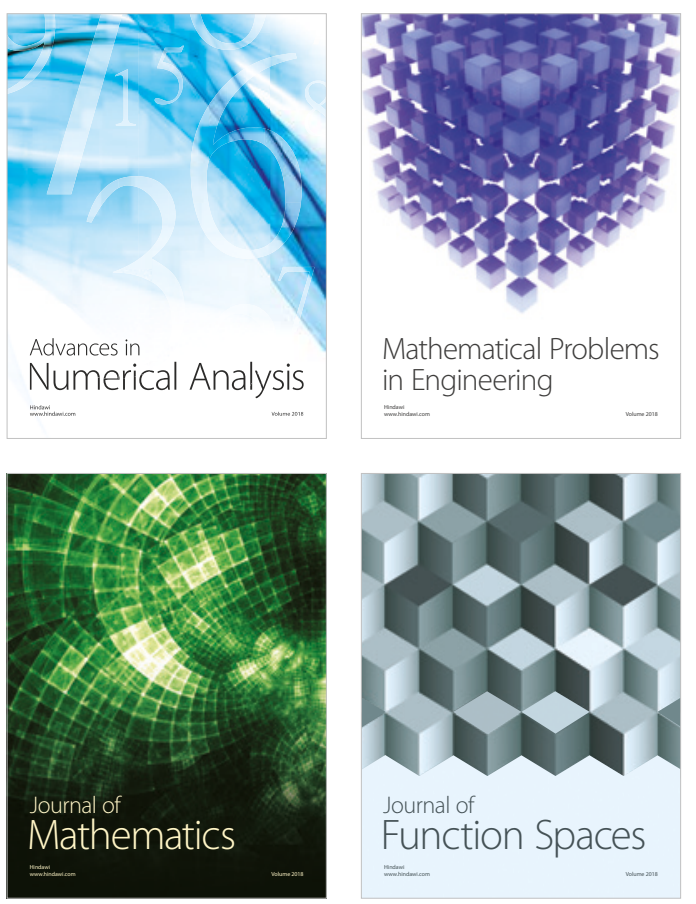

Mathematical Problems in Engineering

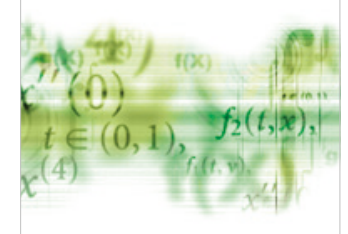

International Journal of

Differential Equations

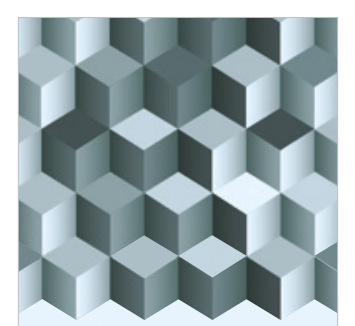

Journal of

Function Spaces
The Scientific

World Journal

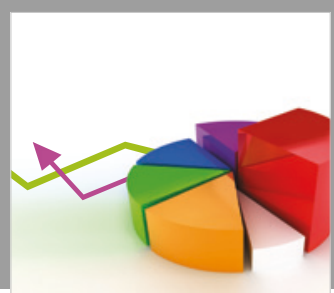

Journal of

Probability and Statistics
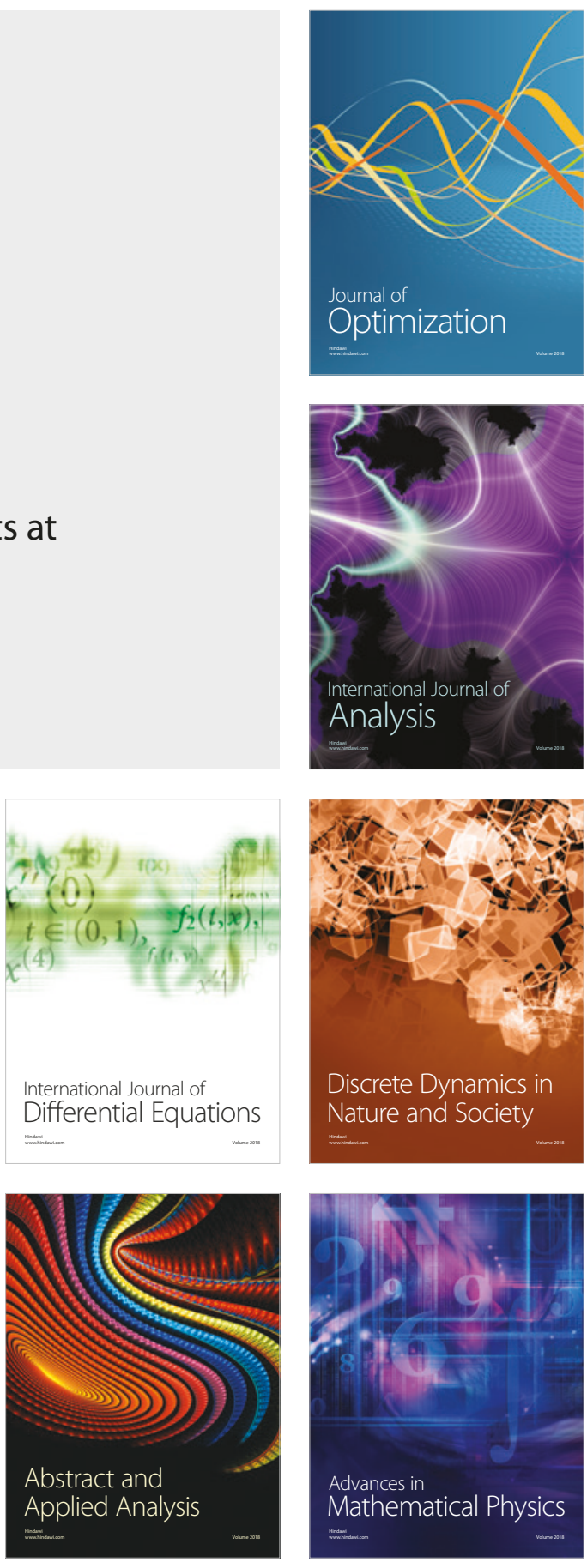